\title{
Capacity Optimization Based on Energy Storage to Restrain Severe Fluctuation of Wind Power
}

\author{
Xiaolin $\mathrm{Fu}^{1}$, Hong Wang ${ }^{1,2}$, Zhijie Wang,*, Yongxin $\mathrm{Li}^{1}$, Jindu $\mathrm{Lv}^{1}$, Ruijiu Jin ${ }^{1}$ \\ 1. Shanghai Dianji University, Shanghai 201306, China \\ 2.University of Strathclyde, Glasgow, UK \\ E-mail:wzjsdstu@163.com (Corresponding Author)
}

\begin{abstract}
In order to solve the coordination problem between the economy and the stabilization effect of energy storage system, a capacity optimization model based on energy storage to suppress the violent fluctuation of wind power is proposed, and a multi-objective function with the maximum wind power dissipation capacity and the minimum operating cost of energy storage system is established. Considering the average annual cost and penalty cost of the whole life cycle, an evaluation index with correlation coefficient as the fitting degree is proposed, and the reference power of wind farm grid connection is optimized by particle swarm algorithm. Using the operation data of Qidong Wind Farm in Jiangsu Province, the theoretical validity is verified, the coordination is improved to the greatest extent, and the capacity demand for energy storage system is reduced.
\end{abstract}

Keywords: Power fluctuation; Energy storage; Capacity optimization.

\section{Introduction}

The additional energy storage system can improve the power fluctuation of wind turbines and improve the power quality and wind power acceptance capacity. However, the economy of energy storage is negatively related to the energy storage capacity. The larger the capacity, the better the stabilization effect of wind power fluctuations and the worse the economy. The balance between energy storage economy and power stabilization effect is the most important reason that restricts the improvement of the energy storage system from the stabilization effect of wind power fluctuations. Therefore, it is necessary to adopt the optimization algorithm and establish a multi-objective capacity optimization model.

\section{Economic analysis}

\subsection{Life cycle average annual cost model}

The life cycle cost of energy storage system refers to the cost of investment, maintenance and replacement of energy storage equipment during its life cycle [1]. Energy storage system mainly includes energy conversion system(PCS) and battery energy management system(BMS)[2]. Therefore, the initial total input cost (TCC) of the energy storage system includes the cost of the power electronic converter (PCS) and the energy management system and the total cost of the energy storage unit. Maintenance costs consist of battery and super capacitor operating and maintenance costs per kilowatt per year. When calculating the replacement cost, the super capacitor is not considered, but only the annual replacement cost of the battery and the annual replacement cost per unit capacity of the battery unit are calculated. Through the above calculation, the average annual cost model for the whole life cycle of the hybrid energy storage system can be finally obtained.

$$
C_{\text {sum }}=A C C+O M C+A R C
$$

In the formula (1), quantities are defined as: $A C C$ is annual initial installation cost of energy storage system; $O M C$ is annual maintenance and maintenance cost of energy storage system; $A R C$ is energy storage system replacement cost.

$$
\begin{aligned}
& T C C=T C C_{b}+T C C_{c} \\
& \left\{\begin{array}{l}
T C C_{b}=C_{i-p} \times P_{b E S S}+C_{i-e} \times \frac{E_{b E S S}}{\eta_{b}} \\
T C C_{c}=C_{j-p} \times P_{C E S S}+C_{j-e} \times \frac{E_{c E S S}}{\eta_{c}}
\end{array}\right.
\end{aligned}
$$

where, $T C C_{b}$ is the initial installation cost of the battery; $T C C_{c}$ is initial Installation Cost of Capacitor; $C_{i-p}, C_{j-p}$ 
are the unit power cost of the battery and the capacitor device is yuan / kW respectively; $P_{b E S S}, P_{c E S S}$ are the powers of that battery and the capacitor device, respectively; $C_{i-p}, C_{i-e}$ are the unit capacity cost of battery and capacitor unit, yuan / kWh respectively; $E_{b E S S}, E_{c E S S}$ are capacities of energy storage device; $\eta_{b}, \eta_{c}$ are the conversion efficiency of that battery and the capacitor device, respectively.

$$
\begin{aligned}
A C C & =T C C \times C R F \\
C R F & =\frac{i_{r}\left(1+i_{r}\right)^{Z}}{\left(1+i_{r}\right)^{Z}-1} \\
A C C & =T C C_{b} \times C R F_{b}+T C C_{c} \times C R F_{c} \\
& =\left(C_{i-p} \times P_{b E S S}+C_{i-e} \times \frac{E_{b E S S}}{\eta_{b}}\right) \times \frac{i_{r b}\left(1+i_{r b}\right)^{Z_{b}}}{\left(1+i_{r b}\right)^{Z_{b-1}}}+\left(C_{j-p} \times P_{C E S S}+C_{j-e} \times \frac{E_{c E S S}}{\eta_{c}}\right) \times \frac{i_{r c}\left(1+i_{r c}\right)^{Z_{c}}}{\left(1+i_{r c}\right)^{Z_{c-1}}}
\end{aligned}
$$

where, ACC is annual initial installation investment cost; $C R F_{b}, C R F_{c}$ are the cost recovery coefficient of that energy storage battery and the super capacitor respectively.

$$
O M C=O M_{b} \times P_{b E S S}+O M_{c} \times P_{c E S S}
$$

where, $O M_{b}, O M_{c}$ are the average annual operating and maintenance cost per kilowatt of battery and super capacitor is yuan / $\mathrm{kW}$ respectively.

$A R C=A \times \frac{E_{b E S S}}{\eta_{b}}$

where, A is average annual replacement cost per unit capacity of battery cell. To sum up, the average annual cost model for the whole life cycle of the energy storage system is:

$C_{s u m}=\left(C_{i-p} \times P_{b E S S}+C_{i-e} \times \frac{E_{b E S S}}{\eta_{b}}\right) \times C R F_{b}+\left(C_{j-p} \times P_{c E S S}+C_{j-e} \times \frac{E_{c E S S}}{\eta_{c}}\right) \times C R F_{c}+O M_{b} \times P_{b E S S}+O M_{c} \times P_{c E S S}+A \times \frac{E_{b E S S}}{\eta_{b}}$

Can be simplified to:

$C_{\text {sum }}=E_{b E S S} \times\left\{\left[\frac{C_{i-p}}{M D D_{b}}+\frac{C_{i-e}}{\eta_{b}}\right] \times C R F_{b}+\frac{A}{\eta_{b}}+\frac{O M_{b}}{M D D_{b}}\right\}+E_{C E S S} \times\left\{\left[\frac{C_{j-p}}{M D D_{c}}+\frac{C_{j-e}}{\eta_{c}}\right] \times C R F_{c}+\frac{O M_{c}}{M D D_{c}}\right\}$

where, $M D D_{b}=E_{b E S S} / P_{b E S S}, M D D_{c}=\mathrm{E}_{\mathrm{cESS}} / \mathrm{P}_{\mathrm{cESS}}$ are the maximum discharge duration of the energy storage battery and the super capacitor respectively; $C R F_{b}, C R F_{c}$ are cost recovery factor, reference to relevant data and practical engineering application.

Therefore, the variables that affect the average annual cost of the energy storage system are the rated capacity and rated power of the energy storage system.

\subsection{Mathematical model of penalty cost}

Due to the limitation of the maximum charge, discharge power and capacity of the energy storage system, it is still difficult to alleviate the power fluctuation in the complex wind power climbing scene after the energy storage system acts, and the resulting loss is called penalty cost. Including the cost of abandoning the wind and the penalty cost of not achieving the expected stabilization effect. Therefore,

$$
\begin{aligned}
& C_{\text {punish }}=C_{\text {up }}+C_{\text {down }} \\
& C_{\text {up }}=\beta \cdot \sum_{t=1}^{T} S_{L O W E}(t)\left\{P_{B}(t) \Delta t-\left[E_{b E S S N}-E_{b E S S}(t-1)\right]\right\}+\beta \cdot \sum_{t=1}^{T} S_{L O W E}(t)\left\{P_{C}(t) \Delta t-\left[E_{c E S S N}-E_{c E S S}(t-1)\right]\right\} \\
& S_{\text {LOWE }}(t)= \begin{cases}1 & P_{\text {BorC }}<0 \text { and } P_{\text {BorC }} \Delta t>E_{\text {borcESSN }}-E_{\text {borcESS }}(t-1) \\
0 & \text { other }\end{cases}
\end{aligned}
$$

where, $P_{B}, P_{C}$ are the throughput power command values of batteries and super capacitors; $E_{b E S S N}, E_{C E S S N}$ are the maximum capacity of that battery and the sup capacitor respectively; $E_{b E S S}(t-1), E_{c E S S}(t-1)$ are the remaining capacity of the battery and the super capacitor at the last moment respectively; $\beta$ is cost coefficient of unit air abandonment capacity; $S_{L O W E}(t)$ is boolean quantity. 


$$
C_{\text {down }}=\alpha \times \sum_{t=1}^{T} S_{\text {LOWE }}(t)\left\{P_{B}(t) \Delta t-\left[E_{b E S S N}-E_{b E S S}(t-1)\right]\right\}+\alpha \times \sum_{t=1}^{T} S_{\text {LOWE }}(t)\left\{P_{C}(t) \Delta t-\left[E_{c E S S N}-E_{c E S S}(t-1)\right]\right\}
$$

where,

$$
S_{\text {LOWE }}(t)= \begin{cases}1 & P_{\text {BorC }}>0 \text { and } P_{\text {Borc }} \Delta t>E_{\text {borcESSN }}-E_{\text {borcESS }}(t-1) \\ 0 & \text { other }\end{cases}
$$

where, $\alpha$ is the operating cost of insufficient energy.

\section{Capacity optimization configuration model and evaluation index}

The objective function is to find the relationship between energy storage economy and stabilization effect, reduce wind abandonment, and improve the grid's acceptance of wind power [3]. The objective function of wind reception capacity $f_{1}$ and the total economic cost of energy storage $f_{2}$ are as follows:

$$
\begin{aligned}
& S^{*}{ }_{\text {LOWE }}(t)= \begin{cases}1 & P_{\text {BorC }}>0 \text { or } P_{\text {BorC }}<0 \text { and } P_{\text {BorC }} \Delta t>E_{\text {borcESSN }}-E_{\text {borcESS }}(t-1) \\
0 & \text { other }\end{cases} \\
& \left\{\begin{array}{l}
\max f_{1}=\left(1-\frac{\sum_{t=1}^{T} S^{*}{ }_{L O W E}(t)\left\{P_{B}(t) \Delta t-\left[E_{b E S S N}-E_{b E S S}(t-1)\right]\right\}+\sum_{t=1}^{T} S^{*}{ }_{L O W E}(t)\left\{P_{C}(t) \Delta t-\left[E_{C E S S N}-E_{C E S S}(t-1)\right]\right\}}{\sum_{t=1}^{T} P_{W}(t) \Delta t}\right) * 100 \% \\
\operatorname{mim} f_{2}=C_{\text {sum }}+C_{\text {punish }}
\end{array}\right.
\end{aligned}
$$

The constraint conditions include the power fluctuation constraint of the combined output of the wind storage system, the power and capacity constraint of the energy storage battery, and the power and capacity constraint of the super capacitor.

By optimizing the particle swarm optimization algorithm, the expected output power satisfying the constraints can be fitted to the predicted power as much as possible [4]. The output of the energy storage device is the difference between the expected output power and the predicted power, so the smaller the difference, the smaller the minimum capacity of the energy storage device and the lower the fixed cost. The expected output objective function is:

$$
P_{\text {refs }}(i)=\min \sum_{i=1}^{T}\left(P_{\text {ref }}(i)-P^{*}(i)\right)^{2}
$$

where, $P_{\text {ref }}$ is Grid - connected Reference Power Satisfying Wind Power Fluctuation Constraints; $P^{*}$ is Predicted wind power; $P_{\text {refs }}$ is Optimized wind power grid-connected reference value.

This paper puts forward the evaluation index of correlation coefficient. The evaluation index can be used to describe the degree of approximation between the reference wind power grid-connected power and the actual wind power stabilized by hybrid energy storage. The formula is as follow:

$$
r=\frac{\sum_{i=1}^{N} \mid P_{O}(i)-\overline{P_{O}} \| P_{\text {refs }}(i)-\overline{P_{\text {refs }} \mid}}{\sqrt{\sum_{i=1}^{N}\left|P_{O}(i)-\overline{P_{O}}\right|^{2}} \sqrt{\sum_{i=1}^{N}\left|P_{\text {refs }}(i)-\overline{P_{\text {refs }}}\right|^{2}}}
$$

where, $P_{O}$ is Wind - storage combined output power; $\overline{P_{O}}$ is Average output power of combined wind and storage; $P_{\text {refs }}$ is Reference value of grid-connected power of wind farm; $\overline{P_{\text {refs }}}$ is Reference average of grid-connected power of wind farm.

\section{Optimal capacity allocation based on particle swarm optimization algorithm}

Particle Swarm Optimization (PSO) can realize the global optimal solution with faster calculation speed and is more suitable for optimization in dynamic and multi-objective optimization environment [5]. The global optimal particle is searched in the solution space by following the current optimal particle.

In a wind farm, a multi-objective function can be changed into a single objective function by adding a weight coefficient to the objective function. The dimension and importance of the function are unified by increasing the weight coefficient. The original multi-objective function can be transformed into a single objective function as follows:

$\min f=\min \left(\lambda\left(1-f_{1}\right)+f_{2}\right)$ 
where, $\lambda$ is weight coefficient.

The specific process of the basic particle swarm algorith [6] is:

1) Initialization of particle swarm, including swarm size v, position $x_{i}$ and speed of each particle.

2) Calculation of fitness value of each particle.

3) Comparing the fitness value of each particle with the individual extreme value, if $F i t(i)>P_{\text {best }}(i)$, so replacing Fit $(i)$ with $P_{\text {best }}(i)$.

4) Comparing the fitness value of each particle with the global extreme value, if $F i t(i)>g_{\text {best }}(i)$,so replacing Fit $(i)$ with $g_{\text {best }}(i)$.

5) Updating the position and speed of particles.

6) Exit if the end condition is met, i.e. the error is good enough or the maximum number of cycles is reached, otherwise return to (3).

\section{Analysis of simulation results of an example}

This example selects the wind power operation data from January 1, 2011 to December 31, 2011 from 0: 00 to 24: 00 in Qidong Wind Farm in Jiangsu Province, and solves the multi-objective function proposed in this paper through particle swarm optimization algorithm, limited to the one-year operation time of the hybrid energy storage system. Fig.1 shows the relationship between the capacity of energy storage systems and their operating costs. Total $\operatorname{cost} f_{2}=C_{\text {sum }}+C_{\text {punish }}$, with the increase of energy storage capacity, $f_{2}$ has a minimum value, and point $\mathrm{A}$ in the figure is the minimum value point. When the energy storage capacity is $25 \mathrm{MWh}$, the total operating cost of the energy storage system is the smallest.

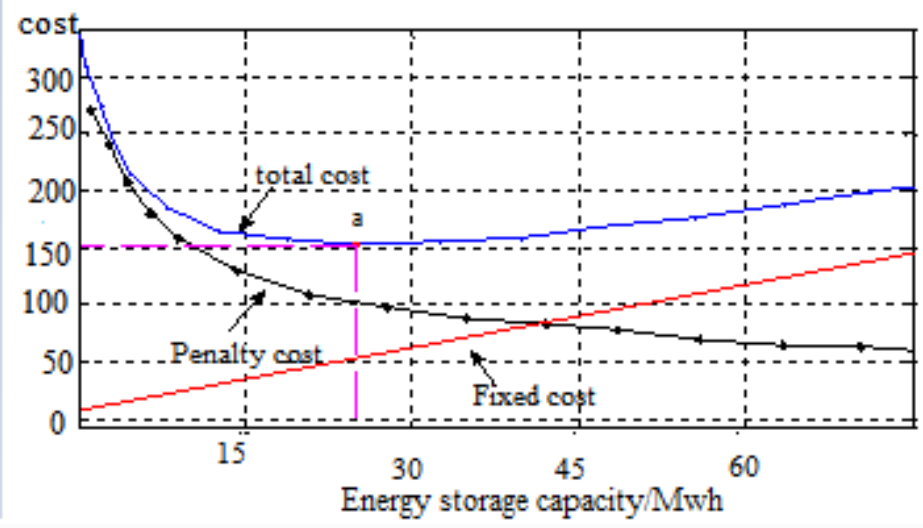

Fig.1 The relationship between the economy and the capacity of the energy storage

As shown in Fig.2, the relationship between wind power reception capacity and energy storage capacity, the larger the energy storage capacity, the stronger the wind power reception capacity, and the higher the energy storage system cost.

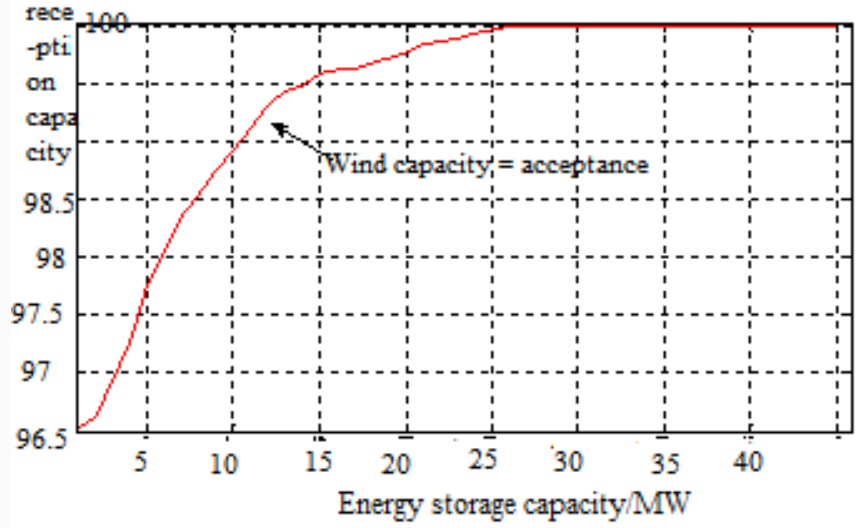

Fig.2 Relationship between wind power and capacity of energy storage 
The purpose of this example optimization algorithm is to find a harmonious relationship between wind energy reception capacity and energy storage economy. Specific optimization results are shown in table 1.

Tab. 1. Capacity optimization of hybrid energy storage system in wind farm

\begin{tabular}{cccccccc}
\hline $\mathrm{E}_{E S S N}$ & $\mathrm{E}_{b E S S N}$ & $\mathrm{E}_{c E S S N}$ & $f_{1}$ & $f_{2}$ & $C_{\text {sum }}$ & $C_{\text {punish }}$ & $r$ \\
\hline 24.8 & 20.12 & 4.68 & $99.6 \%$ & 156.246 & 55.045 & 101.201 & 0.982 \\
\hline
\end{tabular}

According to the optimization results, when the wind farm is equipped with $24.8 \mathrm{MW} / \mathrm{h}$ energy storage capacity, the wind power acceptance capacity is $99.6 \%$, and the total operating cost of the hybrid energy storage system is 156.246. The similarity between the wind power curve after climbing control by the hybrid energy storage system and the expected output wind power reference value is 0.982 .

Fig. 3 shows the relationship between grid-connected power and actual wind power after wind power fluctuation is controlled by energy storage assistant.

Figs. 4 and 5 are diagrams of comparison. The variable is to use energy storage system to assist control wind power fluctuation. Fig. 4 compares the fluctuation rate of wind power in one minute time scale, and Fig.5 compares the fluctuation amplitude of wind power in 30 minutes time scale.

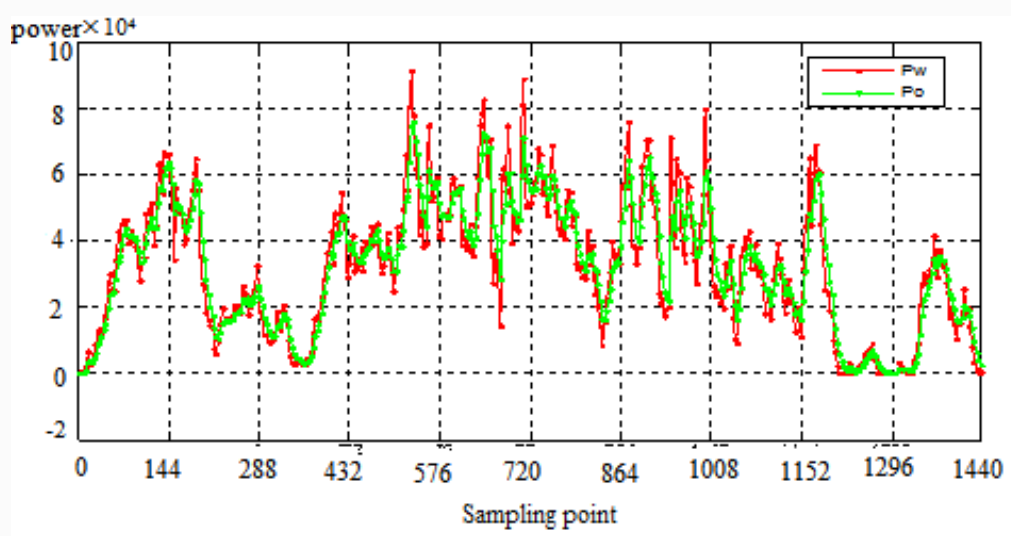

Fig.3 Diagram of wind power and the actual power connected with grid

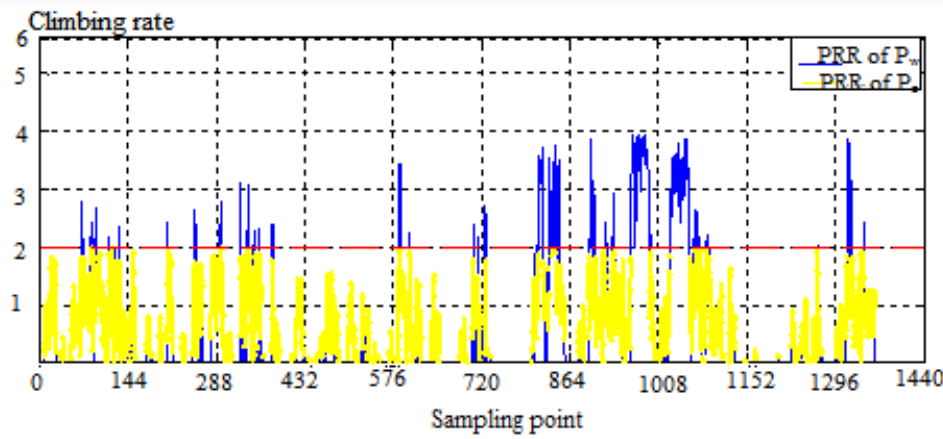

Fig.4 Comparison of PRR before and after optimization

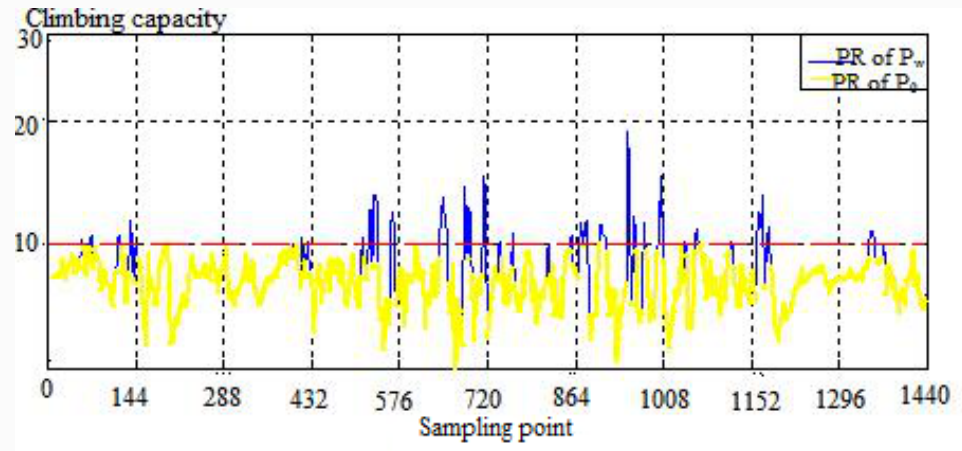

Fig.5 Comparison of PR before and after optimization 
From fig. 3, fig. 4 and fig. 5, it can be seen that hybrid storage system can effectively mitigate wind power fluctuation.

As can be seen from fig. 6, the actual grid-connected power is highly fitted with the optimized reference output power curve, which can reduce the capacity requirement of the energy storage system. However, due to the limitation of charge and discharge power and residual energy state of the energy storage system, air abandonment and overdischarge also resulted in some deviations at points 288, 576, 1008 and 1200.

As shown in fig. 7, the output power curves of the supercapacitor and the battery are respectively controlled by the low-pass filtering algorithm. The low-frequency component is suppressed by the battery, and the highfrequency component is suppressed by the supercapacitor. In fig. 7, it can be seen that insufficient power is suppressed at points 576 and 1008. At points 288 and 1200, the charging power reached its maximum and the phenomenon of wind abandonment occurred.

As shown in fig. 8, the SOC of the battery changes under the control strategy based on the scene switching system. After the scene switching system is activated, the battery does not appear to be deeply charged and discharged in most cases, effectively protecting its service life. However, due to the drastic fluctuation of leeward electric power in extreme weather, the battery tends to be overcharged and over discharged as shown at sampling points 216, 648, 936, etc. in the figure, damaging the battery.

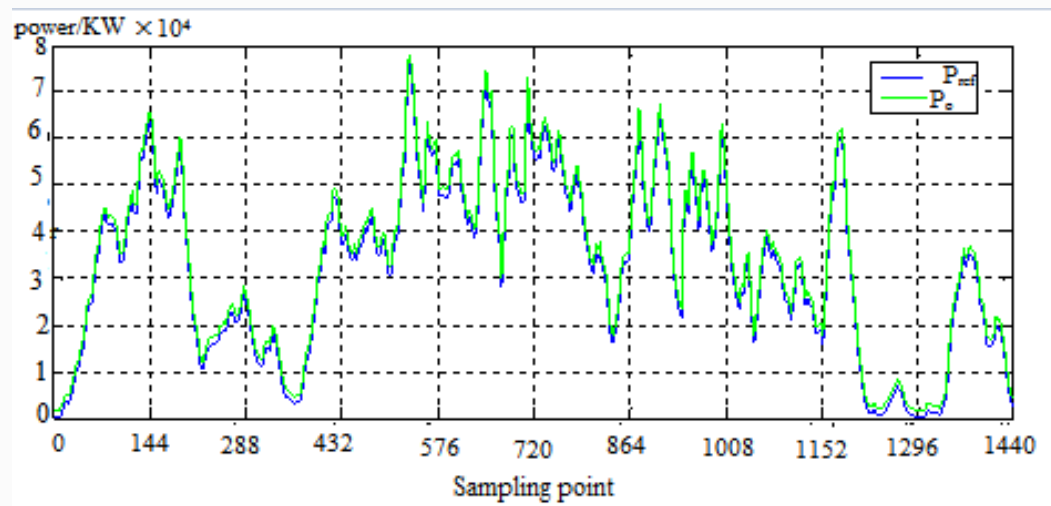

Fig.6 The fitting degree of the actual power connected with the grid and the reference output power

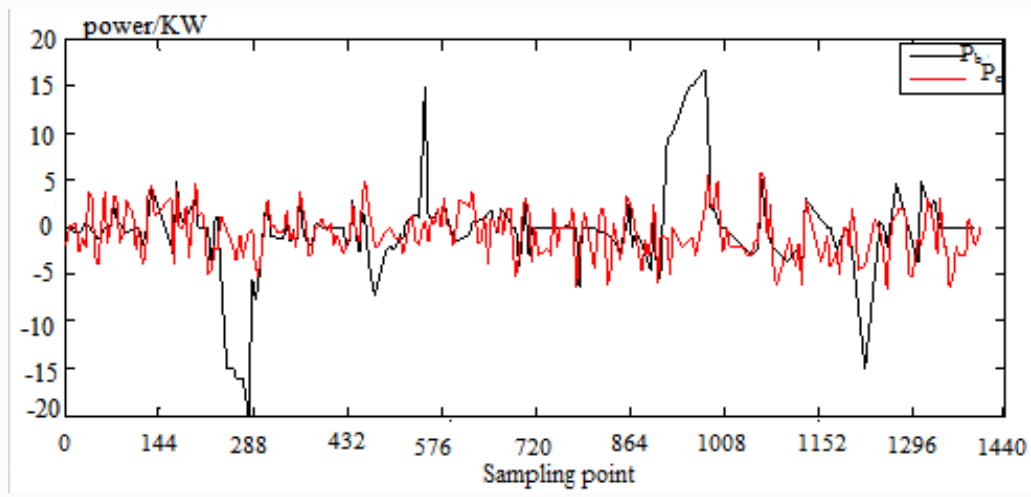

Fig.7 Power allocation of hybrid energy storage system

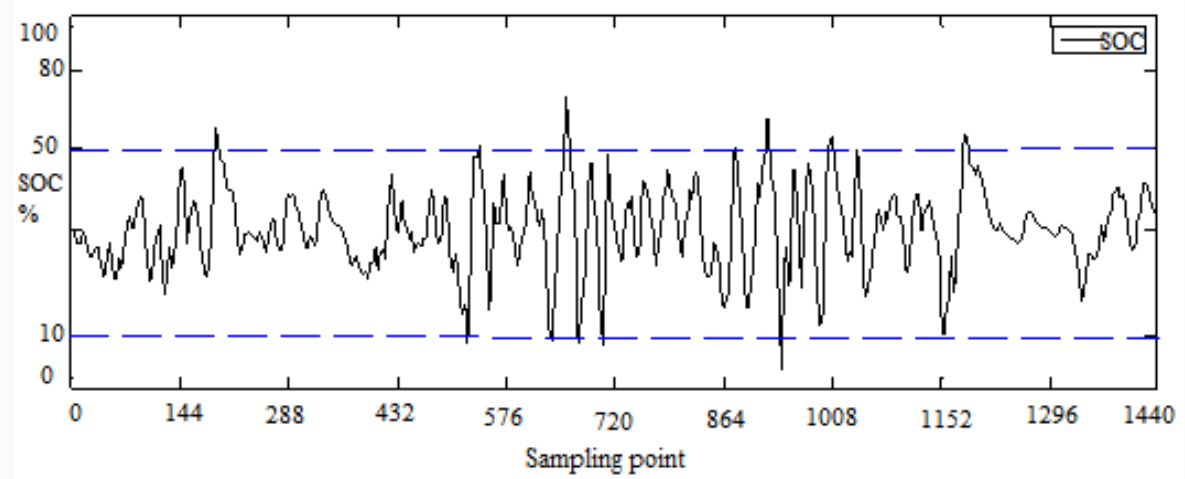

Fig.8 The SOC of battery based on SSA 
Fig. 9 shows the energy state of the battery after the air discarding method is combined, the overcharge phenomenon of the battery is effectively relieved, and the damage to the battery life is reduced.

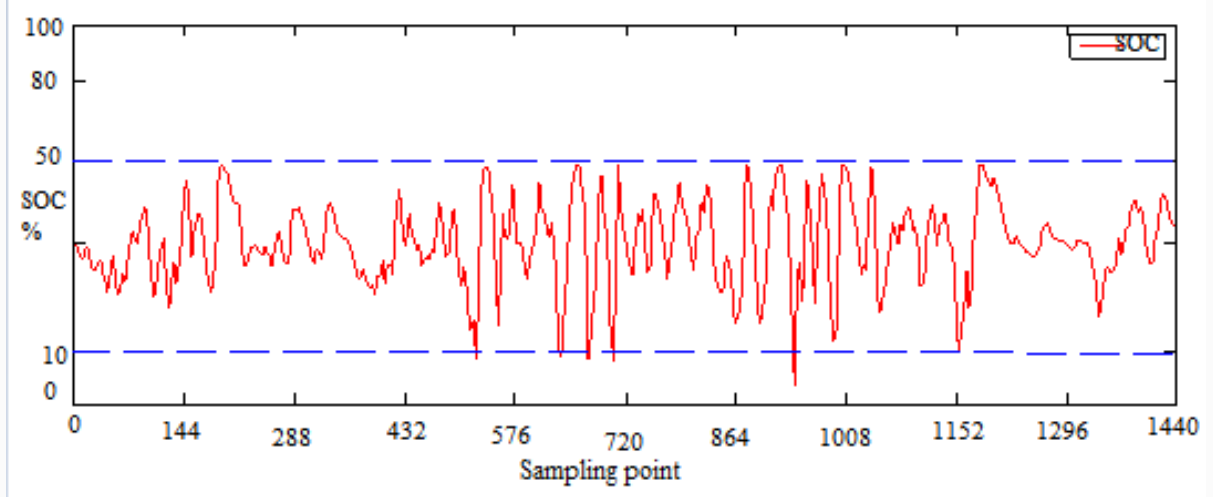

Fig.9 The SOC of battery with the means of abandoned wind

\section{Conclusions}

To sum up, economy and capacity should be considered comprehensively when suppressing wind power fluctuation with energy storage, and the two are not completely independent and cannot be directly related. The optimization algorithm and capacity optimization model proposed in this paper can find out the extreme point of operating cost, reduce the burden of energy storage system to a certain extent, fit the actual power, and achieve the coordination of wind power acceptance capacity and energy storage system economy.

\section{Acknowledgments:}

This work is supported by the National Natural Science Foundation of China (Grant Nos.51477099); Key Laboratory of Control of Power Transmission and Conversion (SJTU), Ministry of Education (2016AB14).

\section{References}

[1] Xiang Yupeng, Wei Zhinong, Sun Guoqiang, Sun Yonghui, Shen Haiping. Optimal Configuration of Battery Energy Storage System in Distribution Network Based on Life Cycle Cost.Power Grid Technology. 2015;01:264 $-270$.

[2] Wang Zhejun. Research on Mobile Energy Storage Power Station. Anhui: Anhui University of Technology. 2018.p.1 - 6.

[3] Yang Jianbai. Research on Optimal Configuration Strategy of Power Grid Energy Storage System. Qingdao: Qingdao University of Science and Technology; 2018.p. 25 - 26.

[4] Zhao Yan. Research on Short - term Wind Speed and Power Forecast Technology for Wind Farms. Tianjin: Tianjin University of Technology; 2018.p.27 - 29.

[5] Tang Kezong, Li Huiying. An Improved Particle Swarm Optimization Algorithm for Solving Complex Optimization Problems. Nanjing: journal of nanjing university of science and technology;2015.

[6] Xie Fei, Research on Optimization of Power Supply Capacity Allocation in Multi - energy Industrial Parks Based on Hybrid Particle Swarm Optimization. Henan: North China University of Water Resources and Hydropower; 2018.p.21 - 23. 